\title{
Unplanned Readmission to Hospitals at Home Care Set up in India - A Comparative Study
}

\author{
Punitha Singh*, Gaurav Thukral, Joseph PC, Babusona Sahoo \\ Department of Nursing and Clinical Governance, Heath Care at Home India Pvt Ltd, Noida, India
}

Email address:

punitha.singh@hcah.in (P. Singh)

${ }^{*}$ Corresponding author

\section{To cite this article:}

Punitha Singh, Gaurav Thukral, Joseph PC, Babusona Sahoo. Unplanned Readmission to Hospitals at Home Care Set up in India - A Comparative Study. American Journal of Nursing Science. Vol. 8, No. 6, 2019, pp. 356-360. doi: 10.11648/j.ajns.20190806.21

Received: November 11, 2019; Accepted: December 12, 2019; Published: December 26, 2019

\begin{abstract}
The health care delivery system has undergone dramatic shifts in care settings in the recent past. More patients are receiving professional home care following discharge from hospitals, skilled-care facilities and rehabilitation centers. Home care has become an integral part of patient recovery especially among the long stay patients. Skilled nursing care delivered in the patient's home may prevent, foresee or limit costly readmissions back to hospitals. There are few other adverse events which are likely to happen at hospital settings like patient fall, developing HAI etc can be totally prevented in a home environment by one to one care which unloads the financial burden on both sides. There have been number of studies undertaken and many literatures are available on hospital readmission rates for reference. Nonetheless not much focus has been given to this particular area i.e. readmission of patients to hospitals while they are being taken care at home settings. Hence this study helps in understanding the readmission rates in home-based care. The study duration was from Jan 2018 - June 2018 to Jan 2019- June 2019. This was a comparative study and the study group was critically ill patients who were admitted during this time at Health care at home $(\mathrm{HCAH})$, India.
\end{abstract}

Keywords: Un Planned re Admissions, Comparative Study, Critical Care Patients Distribution, Causes of re Admission

\section{Introduction}

Health Care at home India is a leading home health care service provider in India. HCAH follows the best practices in clinical care and maintains international standards and protocols for care delivery. [1] HCAH maintains high standards in patient satisfaction and the net promoter's score has been consistently high. It provides a plethora of services including ICU at home, general nursing care, physio therapy services and various other clinical services at home. Scope of each service is defined according to regulatory and statutory requirement of the country [2].

The vision of HCAH is strive to be the most people-centric, credible and comprehensive home healthcare solution provider in India.

The mission of HCAH is to fulfill the vision by creating a service delivery model with "people centricity" at the core of it. Delivering credible clinical outcomes, for every patient, every time. Evolving a scalable and self-sustaining business model.
Key words: HCAH (Health Care at Home), CRCC (Critical care service code), ICU at home (Intensive care unit at home), unplanned re admission, CET (Clinical Evaluation Team), MEWS (Modified Early Warning Signs), PCS (patient care system).

Objectives of the Study;

To explore the Readmission status.

To Identify the most common causes for unplanned readmission.

To reduce the readmission rates.

To improve care delivery by competent professionals.

Assumptions:

This is assumed that, Health care at Home India, has lesser percentage of Unplanned re admissions.

Also, it is assumed that, patients hospitalization were done due to severe illness or any kind of situation which is unmanageable at home.

Back ground of the study:

Health care at home is specialized in taking care of critical care patients by setting up ICUs at home and steps the patients 
down gradually at home environment depending on the prognosis. During the process of care being rendered to patients at home, it was observed that the re admission rates to the hospital were high and the reasons identified were many [3]. However, some of those readmissions could have been avoided easily if adequate attention was given by the care providers [4]. This comparative study would clearly highlight how the readmission rates were brought down during similar period of the following year after adequate training and frequent audits.

Some of the top reasons [5] for unplanned readmission of the patients during the study period from January 2018- June 2018 which were analyzed by an expert team at the business support center were:

1. Tachycardia.

2. Tachypnoea.

3. Dyspnea/shortness of breath leading to desaturation.

4. Hypo or hypertension.

5. Abdominal distention.

6. High grade fever.

7. Ascites.

8. UTI.

9. Deranged or abnormal lab values.

Hospital readmission did not make sense to any of the stake holders at home health care to admit the patients back to hospitals for some of the above-mentioned reasons which can be handled by trained professionals in most of the cases. We at HCAH realized that some of the conditions related to vital parameters could have been managed well at home if these were identified early. Thus, we worked towards reducing the burden of patients getting readmitted to the hospital using combinations of various methods which helped us to improve our overall service delivery and reduced hospital readmissions. Many a times families sought home care only because they did not want to keep their loved ones in the hospital environment for a very reason to be with them. [6]

\section{Method of Study}

A comparative study to look into the unplanned readmissions to hospitals in home care set up at HCAH India during similar period between 2018-2019 i.e. from January to June.

\section{Graphical Representation of the Study Conducted}

A comparative study had been conducted at Health care at home India, on patients who got admitted for critical care services on their unplanned readmission to hospitals.

Collected data was analyzed under the following headlines, where.

Table 1. Percentage wise distributions of the Critical Care Patients.

\begin{tabular}{llll}
\hline Year & Month & \% of CRCC Patients & \% of Other Patients admitted to HCAH \\
\hline 2018 & January to June & $28.13 \%$ & $71.87 \%$ \\
2019 & January to June & $36.89 \%$ & $63.11 \%$ \\
\hline
\end{tabular}

Explanation: Data of table no 1 shows that CRCC (critical care) category patients were $28.13 \%$ during the study period between January to June 2018 and it was increased to $36.89 \%$ during the study period of January to June for the year of 2019. This is mainly due to general awareness among the public and increasing promotion of Home Health Care services at India by providing world class services.

Table 2. Percentage wise distributions of the readmission status of CRCC patients at 2018.

\begin{tabular}{lll}
\hline Month of 2018 & $\begin{array}{l}\text { CRCC patient } \\
\text { percentage }\end{array}$ & $\begin{array}{l}\text { Readmission } \\
\text { Percentages }\end{array}$ \\
\hline January & $30.69 \%$ & $21.21 \%$ \\
February & $26.90 \%$ & $30.18 \%$ \\
March & $26.69 \%$ & $27.11 \%$ \\
April & $31.74 \%$ & $13.69 \%$ \\
May & $26.89 \%$ & $23.43 \%$ \\
June & $25.78 \%$ & $13.78 \%$ \\
Average & $28.13 \%$ & $21.56 \%$ \\
\hline
\end{tabular}

Explanations Table no. 2 explains that the critical care patient's distribution percentages on an average for 6 months period ie. from January to June 2018 was $28.13 \%$ and the unplanned readmissions among these patients were $21.56 \%$, which we at HCAH felt, was high against the international benchmark.
Table 3. Percentage wise distributions of the readmission status of CRCC patients at 2019.

\begin{tabular}{lll}
\hline Month of 2019 & $\begin{array}{l}\text { CRCC patient } \\
\text { percentage }\end{array}$ & $\begin{array}{l}\text { Readmission } \\
\text { Percentages }\end{array}$ \\
\hline January & $33.65 \%$ & $11.59 \%$ \\
February & $39.61 \%$ & $7.31 \%$ \\
March & $40.46 \%$ & $11.49 \%$ \\
April & $38.13 \%$ & $4.87 \%$ \\
May & $37.62 \%$ & $7.59 \%$ \\
June & $31.28 \%$ & $13.11 \%$ \\
Average & $36.89 \%$ & $9.32 \%$ \\
\hline
\end{tabular}

Explanation: During the study period of January 2019 to June2019 the admission percentage of critical care patients have gone up to $36.89 \%$ against the 2018 data during the same time period. However, with all actionable implemented and with stringent monitoring, the readmission has drastically come down to $9.32 \%$ which is well below the international bench mark.

Table 4. Comparisons of Critical care patient's readmission status of the year 2018 and 2019.

\begin{tabular}{lll}
\hline Month & $\mathbf{2 0 1 8}$ & $\mathbf{2 0 1 9}$ \\
\hline January & 21.21 & 11.59 \\
February & 30.18 & 7.31 \\
March & 27.11 & 11.49 \\
April & 13.69 & 4.87 \\
May & 23.43 & 7.59 \\
\hline
\end{tabular}




\begin{tabular}{lll}
\hline Month & $\mathbf{2 0 1 8}$ & $\mathbf{2 0 1 9}$ \\
\hline June & 13.78 & 13.11 \\
Mean & 21.56 & 9.32 \\
\hline
\end{tabular}

Most of the studies (from different countries) have given us a brief idea about "International Benchmark on unplanned
Readmissions" [2]. It ranges anywhere between 11-30\% according to these studies. However, one such study has given us the readmission percentage as $16.4 \%$ and that has been kept as benchmark for our study. Unfortunately, we do not have clear data or defined international bench mark.

\section{Results}

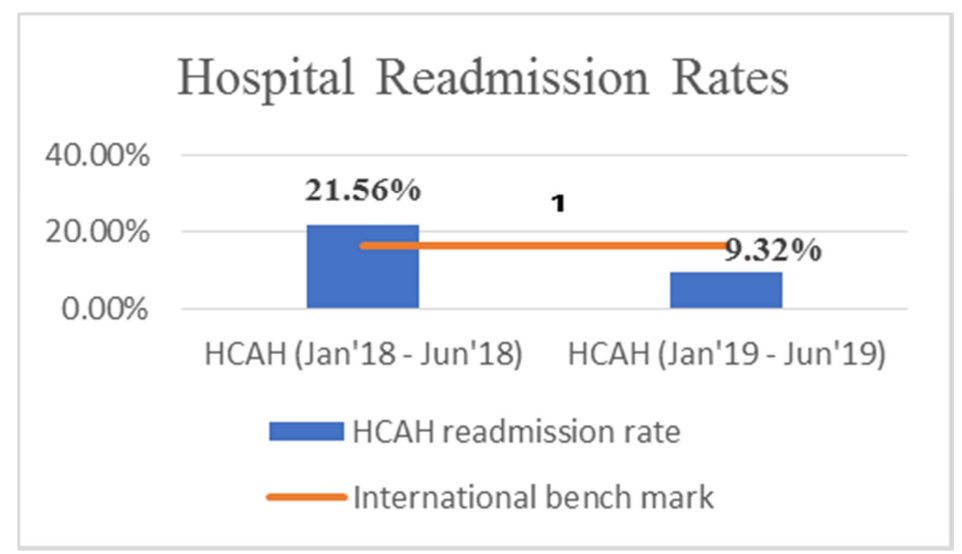

Figure 1. Comparison of readmission rates for the year 2018 and 2019 with international bench mark.

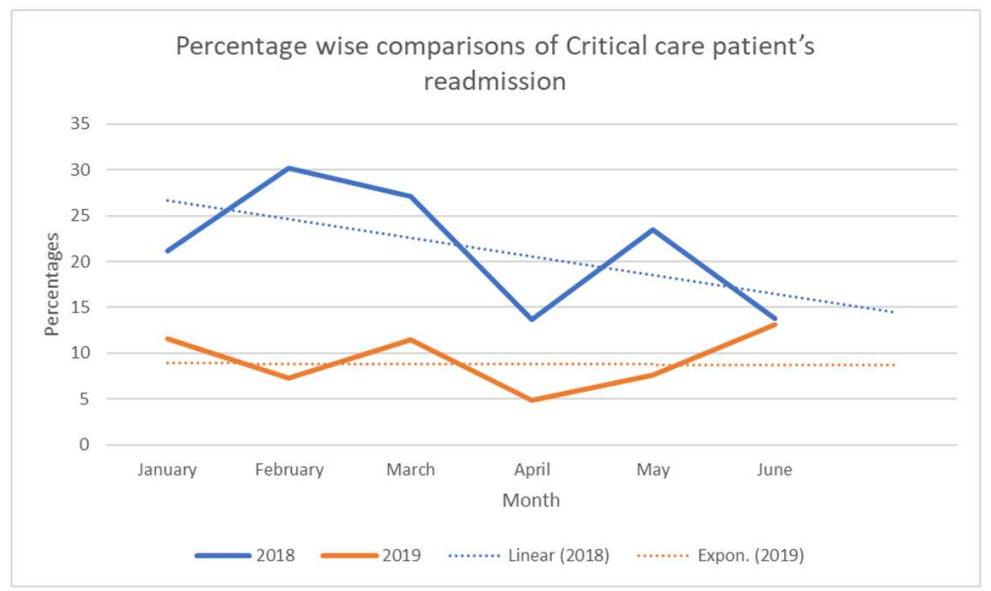

Figure 2. Comparisons of critical care patients readmission status of both years during the study period.

\section{Discussions}

Typically, clients were referred for their first home care admission after few days of hospital stay either for ICU care at home or palliative care or post-surgical care. [7] During their admission with home care, we have had patients who were readmitted to the hospital, usually due to the development of a new problem, or due to deterioration in health status related to the primary or to a secondary medical diagnosis. Significant respiratory, cardiovascular, neurological or GI symptoms were generally present at hospital readmission. Most of the readmitted patients were moderately to critically ill, and most likely in need of acute care. In several occasions' families were not happy about the readmission as they did not want their loved ones to be away from them yet again. When we received such feedback frequently form the families a team started analyzing the reasons and some of the readmissions could have been wisely averted if the nurses at home environment were more attentive. As mentioned in the background of the study, the reasons for readmission were analyzed critically and decided to work around it to avoid some of the causes for the same.

Our focus was on the following to start with:

1. An expert team analyzing the vital parameters of the patient.

2. Technology enabled MEWS (Modified Early Warning Signs) for alerts.

3. Proper training on handling emergencies.

4. Appropriate deployment of staff, based on their competency.

5. Validation by the managers before any case was referred for further management.

Once the process for this exclusive effort was in place, a task force was created in each of our units across the country and trained on the focus points. Every unplanned readmission 
to the hospital was made as part of incident reporting and proper Route Cause Analysis and CAPA (Corrective action and preventive action) were done. The documentations were analyzed by the Clinical Evaluation Team (CET) centrally to see if the hospitalization could have been avoided. This continued for 6 months and we could see remarkable results in reduction of unplanned readmission.

MEWS (Modified Early Warning Signs) [8] were incorporated in PCS (Patient Care System)-a mobile app for the nurses to enter vital signs. Hence abnormal readings were highlighted, and the staff were alerted immediately for further observation.

Escalation matrix [9] was in place for reporting any such untoward events at home environment that could prompt the reporting manger and to seek his/ her advice for further management.

Once these were implemented, it was observed that the number of unplanned readmissions to the hospitals from home [10] were considerably reduced. Preventable conditions such as tachypnoea, tachycardia, dyspnea leading to desaturation, hypo tension etc were largely avoided.

Patients' readmission were mainly for the following reasons during the same period in 2019 from January to June:

1. Convulsion.

2. Oozing from surgical wound.

3. Shortness of Breath (SOB).

4. Deranged electrolytes.

5. Drowsiness.

6. Severe abdominal pain.

7. High grade fever.

8. Internal bleeding.

Careful observation of patients' vital status, appropriate timely escalation, and seeking medical support when needed, [11] have brought down the percentages of hospital readmission which is considered to be a huge financial burden

[12] and discomfort to the patients and their families.

\section{Limitation \& Supports}

Inclusion:

This study is completely limited to the organization "Health care at Home India", and its nursing staff. It is limited to "CRCC 4, CRCC3, CRCC2 and CRCC1" (who are critically ill) category patients only.

\section{Exclusion:}

This study excluded all "Step Down" category patients and other Home health care service providers of the organization.

Support:

Clinical Evaluation Team (CET)" of Health care at Home India was a huge support in collecting, collating and analyzing data for this study. Senior management of organization has been a tremendous support throughout this study.

\section{Conclusion of the Study}

This study was limited only to critical care patients of HCAH. There could have been a different data derived if re admission numbers were calculated for all patients who were admitted in $\mathrm{HCAH}$ for home care. However, this gave an insight that many readmissions can be prevented if patient monitoring is done and documented appropriately and timely. Staff competency and escalation matrix in place has helped us to reduce the readmission considerably when data were compared between 2 study periods. There is still scope for more effective study [13] with details including age [14], gender, disease conditions, readmissions among over all patients admitted to HCAH etc. Adequate training and improving staff knowledge on critical information has certainly prevented incidences of unplanned readmissions to the hospitals.

It is also very clear that hospital readmission rates can be significantly reduced at home care environment [15] by $1: 1$ staffing and careful monitoring of critically ill patients.

\section{Acknowledgements}

I would like to acknowledge the management of $\mathrm{HCAH}$ who has always been a support and encouraged the team to perform our best.

The nurses at the bedside, who have proved once again that they are decisive and empowered group of professionals who can handle emergency situations well and avoid further re admission.

The Central Evaluation Team for collecting and collating all the relevant data needed for this article.

\section{References}

[1] Health care at home India, L1-06 Nursing manual V1, Pt monitoring pg. 10-14.

[2] Health care at home India, L1-06 Nursing manual V1, Procedure - Care of the ventilated patient, pg. 24-26.

[3] U. S. Department of Veterans Affairs, "Risk prediction models for hospital readmission: A systemic Review". Health Services Research.

[4] D. Butin, "Reducing re admission Tool kit, "Northeast Health Services for Secure".

[5] J. Herrin, S. Andre, K. Kenward, M. Joshi, AM Audel, "Community factors and hospital readmission rates". Health Serv Res. 2015 Feb; 50 (1): 20-39. Vol. 10.1111/1475-6773.12177. Epub 2014 Apr 9.

[6] K. Heery, C. Hull," Ending Hospital Readmissions: A Blueprint for Homecare Providers". vol. 1st edition. pp. 2-10.

[7] P Donovan, "Bench marks in Reducing Hospital readmission", J Salamon, UK.

[8] Prakash Shastri, ICU Manual for nurses under the aegis of Indian Society of Critical Care medicice, 1st edition 2017.

[9] Health care at home India, L1-05 Nursing manual V2, Vital Monitoring, pg. 50-59.

[10] https://www.ncbi.nlm.nih.gov/pubmed/16629969 Readmissions: a primary care examination of reasons for readmission of older people and possible readmission risk factors. 
[11] https://www.ncbi.nlm.nih.gov/pubmed/10571497 Unplanned hospital readmissions: a home care perspective.

[12] G Lehman, "Horizons, United Healthcare". Vol. 1AZ, pp 23-25, April 2019.

[13] https://www.ncbi.nlm.nih.gov/pubmed/8919749 Hospital readmissions during home care: a pilot study.
[14] https://www.ncbi.nlm.nih.gov/pubmed/8990610 unplanned readmissions of elderly patients.

[15] https://homehealthcarenews.com/2018/10/home-health-quality -on-the-upswing-readmission-rates-hold-steady/. 